\title{
Etude Comparative de Deux Garnitures (Noix de Pain de Singe et de Jujube) Dans une Colonne Garnie: Hydrodynamique et Transfert de Matière
}

\author{
Faye Mamadou, \\ Sambe Falilou Mbacké, \\ Toure Alpha Ousmane, \\ Diop El Hadji Moussa, \\ Thiam Aboubacry, \\ Diop Mar Codou Guèye,
}

Laboratoire d'Electrochimie et des Procédés Membranaires (LEPM),

Ecole Supérieure Polytechnique (ESP),

Université Cheikh Anta Diop de Dakar (UCAD), Dakar-Fann, Sénégal

Doi: 10.19044/esj.2019.v15n3p372 URL:http://dx.doi.org/10.19044/esj.2019.v15n3p372

Résumé

Pour bien comprendre l'efficacité de transfert de matière dans les biofiltres, les paramètres hydrodynamiques et de transfert de matière ont été étudiés pour deux garnitures différentes (noix de pain de singe et de jujube) dans un réacteur triphasé à lit fixe fonctionnant à co-courant gaz-liquide. Dans le cadre de ce travail, après la caractérisation des graines une étude d'hydrodynamique et de transfert de matière de l'oxygène a été faite. Les expériences ont été réalisées au sein d'une colonne en verre cylindrique de $0,1 \mathrm{~m}$ de diamètre et $1,5 \mathrm{~m}$ de hauteur. Cette dernière a été remplie avec des noix de pain de singe ou de jujube comme matériaux de garnissage. Les résultats obtenus ont montré que les pertes d'énergie ou la chute de pression $(\Delta \mathrm{P})$ est 3,1875 cmCE contre $0,725 \mathrm{cmCE}$ en moyenne respectivement pour le pain de singe et le jujube, la rétention liquide dynamique $\left(\varepsilon_{\mathrm{LD}}\right)$ est 0,223 contre 0,348 en moyenne respectivement pour le pain de singe et le jujube ainsi que la capacité d'oxygénation (CO) est $70,282 \mathrm{~g} \cdot \mathrm{m}^{-3} \cdot \mathrm{h}^{-1}$ contre 163,674 g. $\mathrm{m}^{-3} \cdot \mathrm{h}^{-1}$ en moyenne respectivement pour le pain de singe et le jujube. Au vue de ces résultats, les noix de jujube présentent sur tous les plans, les meilleures caractéristiques pour être utilisés comme matériaux de garnissage en vue des études pilotes ultérieures.

Mots clés: Matériaux, colonne, hydrodynamique, transfert de matière, oxygène 


\title{
Comparative Study of Two Fillings (Monkey Bread and Jujube Nuts) in a Garnished Column: Hydrodynamic and Mass Transfer
}

\author{
Faye Mamadou, \\ Sambe Falilou Mbacké, \\ Toure Alpha Ousmane, \\ Diop El Hadji Moussa, \\ Thiam Aboubacry, \\ Diop Mar Codou Guèye, \\ Laboratoire d'Electrochimie et des Procédés Membranaires (LEPM), \\ Ecole Supérieure Polytechnique (ESP), \\ Université Cheikh Anta Diop de Dakar (UCAD), Dakar-Fann, Sénégal
}

\begin{abstract}
To fully understand the mass transfer efficiency in biofilters, hydrodynamics and mass transfer parameters were investigated for two different fillings (monkey bread and jujube) in a three-phase fixed-bed reactor operating at co-flow gas-liquid. As part of this work, after the characterization of seeds a hydrodynamic study and transfer of oxygen mass were made. The experiments were carried out in a cylindrical glass column $0.1 \mathrm{~m}$ in diameter and $1.5 \mathrm{~m}$ in height. The latter was filled with monkey bread or jujube as filling materials. The results obtained showed that the energy losses or the pressure drop $(\Delta \mathrm{P})$ is $3.11875 \mathrm{cmCE}$ against 0.725 $\mathrm{cmCE}$ on average respectively for monkey bread and jujube, the dynamic liquid retention $\left(\varepsilon_{\mathrm{LD}}\right)$ is 0.223 against 0.348 on average respectively for monkey bread and jujube and the oxygenation capacity (OC) is $70.282 \mathrm{gm}^{-}$ ${ }^{3} \cdot \mathrm{h}^{-1}$ against $163.674 \mathrm{gm}^{-3} \cdot \mathrm{h}^{-1}$ on average respectively for monkey bread and jujube. As a result, jujube nuts have the best characteristics on all levels for use as packing materials for pilot studies.
\end{abstract}

Keywords: Materials, column, hydrodynamic, mass transfer, oxygen

\section{Introduction}

Les réacteurs à lit fixe généralement appelés bioréacteurs, sont principalement utilisés dans le traitement aérobie des eaux usées urbaines pour l'élimination du carbone, de l'azote et des particules en suspension 
(Sharma et al., 2018). En outre, ils peuvent être utilisés aussi, en vue de l'élimination des métaux lourds dans les eaux potables. Les bioréacteurs combinent compacité et efficacité d'enlèvement élevée. C'est un procédé à biomasse fixée qui permet de réaliser dans le même réacteur l'épuration biologique et la clarification des eaux (Sharma et al., 2018). Les microorganismes se développent sur un matériau support (argile cuite, schistes, pouzzolane, charbons, billes de polystyrène...). Elles se développent sur les parois extérieures des matériaux. Ainsi, afin d'améliorer la conception des bioréacteurs, il est nécessaire de comprendre l'effet des caractéristiques solides sur l'hydrodynamique et le transfert de matière de l'oxygène (Maldonado et al., 2008). La caractérisation de l'hydrodynamique des réacteurs à lit fixe commence par la détermination de la chute de pression du liquide à travers le lit, l'approche proposée par certains auteurs (Park et al., 2018; Pavlišič et al., 2018) explique les tendances observées pour différentes conditions de fonctionnement pour différents types de solides. En effet, les pertes de charges sont mesurées à l'aide d'un manomètre différentiel dans cette étude. En ce qui concerne la rétention liquide dynamique, de nombreux travaux expérimentaux ont été réalisés pour déterminer la rétention liquide dans les réacteurs à lit fixe. Différents auteurs (Orgue, 2012; Maldonado, 2005) ont déterminé la rétention liquide dynamique pour différents types de solides. Malgré les différents solides, leurs données ont montré que la rétention augmentait avec l'augmentation de la vitesse du gaz superficiel et diminuait avec l'augmentation de la vitesse du liquide superficiel (Besagni and Inzoli, 2017; Parisien et al., 2017; Thobie et al., 2017; Zhang et al., 2017). Pour le transfert de matière, les auteurs (Jokiel et al., 2017; Pan et al., 2018) ont déterminé le transfert de matière d'oxygène pour l'eau dans les bioréacteurs en utilisant une méthode basée sur l'oxydation du sulfite en excès suivie de la mesure de la fraction d'oxygène dans le gaz de sortie du réacteur. J.G. Garcia Maldonado a comparé le coefficient de transfert de masse volumique obtenu par un bilan matière réalisé sur la phase gazeuse avec celui obtenu par un bilan matière réalisé sur la phase liquide (Dejaloud et al., 2018; Garcia-Ochoa and Gomez, 2009; Palmerín-Carreño et al., 2016). De surcroit, Différentes méthodes expérimentales ont été utilisées pour définir la caractérisation de l'hydrodynamique du liquide et du gaz des réacteurs à lit fixe. Ces méthodes ont été appliquées pour chaque type de solide et à différentes conditions de fonctionnement du débit de gaz et de liquide (Jiang et al., 2017; Xu et al., 2017).

Bien que de nombreux articles traitent différentes méthodes nécessaires pour caractériser l'hydrodynamique et le transfert de matière dans un réacteur à lit fixe à co-courant, aucun article n'a développé d'explication physique des performances hydrodynamiques et de transfert de matière pouvant aboutir à des résultats fixes. D'où l'intérêt de faire des essais pilote 
afin de déterminer les conditions optimales de marche de chaque colonne spécifique. Par conséquent, l'objectif de cet article est d'évaluer l'effet des caractéristiques solides (matériaux) sur les paramètres hydrodynamiques et de transfert de matière de l'oxygène.

C'est dans ce contexte que nous initions l'étude sur un nouveau type de matériau comme garnissage. Il s'agit des noix de pain de singe et de jujube. Ces derniers sont des produits locaux accessibles, d'où la pertinence de l'initiative. L'enjeu de cette initiative réside dans le sens de valoriser ces produits locaux, qui sont souvent rejetés dans la nature. Après avoir fait la caractérisation des noix de pain de singe et de jujube, ce travail portera sur la détermination du comportement hydrodynamique de la colonne garnie. Ces résultats permettront de faire une étude comparative générale de ces deux matériaux afin de déterminer le matériau qui présente les meilleures caractéristiques.

\section{Matériel et méthodes}

\section{Caractérisation des noix de pain de singe et de jujube}

A l'instar des matériaux de garnissage communément utilisés dans les bioréacteurs, qui sont entre autres l'argile cuite, les schistes, la pouzzolane, les charbons, les billes de polystyrène...nous avons utilisé dans le cadre de cette étude deux types de garnitures : les noix de pain de singe et de jujube.

Les noix sont débarrassées de la pulpe et puis lavées pour enlever les traces restantes. Il faut noter que ces types de garnissage sont des produits locaux.

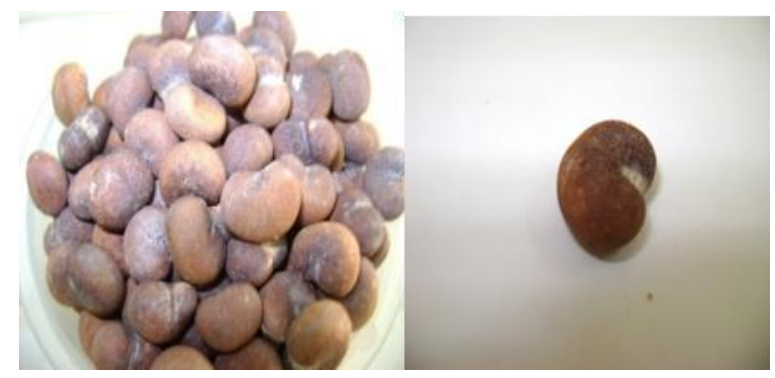

a.

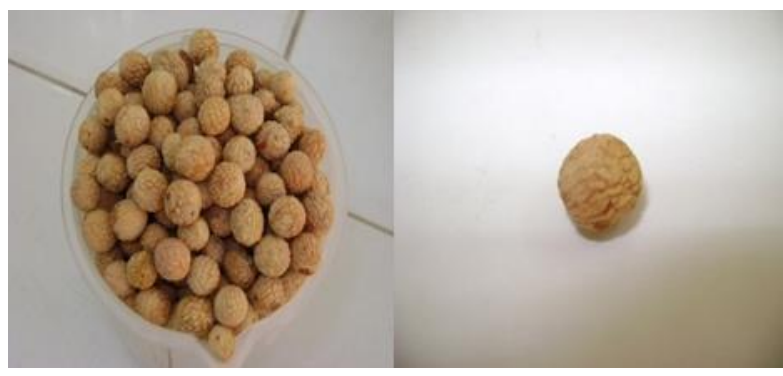

b.

Figure 1 : a. Noix de pain de singe ; b. Noix de jujube

\section{Masse volumique apparente}

Pour la détermination de la masse volumique apparente, nous avons utilisé une éprouvette en verre de $100 \mathrm{~mL}$ et une balance de précision. La balance est tarée avec l'éprouvette graduée puis cette dernière est remplie avec les particules de garnissage (noix de pain de singe ou de jujube) jusqu'au trait de $100 \mathrm{~mL}$ avant de procéder à la pesée. Pour plus de précision 
nous avons effectué trois mesures pour prendre par la suite la moyenne des trois.

La masse volumique est déduite de la relation suivante :

$$
\rho_{\mathrm{ap}}=\frac{\mathrm{m}_{\mathrm{p}}}{\mathrm{V}_{\mathrm{ep}}}
$$

Avec :

$\rho_{\text {ap }}$ : masse volumique apparente

$\mathrm{m}_{\mathrm{p}}:$ masse de pesée

$\mathrm{V}_{\text {ep }}$ : Volume éprouvette $\left(\mathrm{V}_{\mathrm{ep}}=100 \mathrm{~mL}\right)$

\section{Masse volumique réelle}

Pour la détermination de la masse volumique réelle, nous avons utilisé une éprouvette en verre de $100 \mathrm{~mL}$ remplie avec de l'eau jusqu'à $\mathrm{V}_{\mathrm{i}}=50 \mathrm{~mL}$; ensuite une masse de particules bien connue à l'aide de la balance est introduite dans l'éprouvette. Ainsi l'eau initialement introduite augmente de niveau jusqu'à une nouvelle graduation correspondant à un nouveau volume $\mathrm{V}_{\mathrm{f}}$. Le volume des particules $\left(\mathrm{V}_{\mathrm{p}}\right)$ correspond à la différence de volume $\mathrm{V}_{\mathrm{f}}$ $\mathrm{V}_{\mathrm{i}}$.

La masse volumique réelle $\left(\rho_{\mathrm{r}}\right)$ est exprimée par :

\section{Porosité}

$$
\rho_{\mathrm{r}}=\frac{\mathrm{m}_{\mathrm{p}}}{\mathrm{V}_{\mathrm{p}}}=\frac{\mathrm{m}_{\mathrm{p}}}{V_{f}-V_{i}}
$$

Pour déterminer la porosité nous avons utilisé deux éprouvettes de $1000 \mathrm{~mL}$. La première est remplie avec de l'eau jusqu'à $1000 \mathrm{~mL}$ et la deuxième avec les noix de pain de singe ou de jujube jusqu'à $1000 \mathrm{~mL}$. Le protocole consiste à verser l'eau contenue de la première éprouvette dans la deuxième jusqu'au trait de $1000 \mathrm{~mL}$ de sorte que toutes les pores soient remplies d'eau. Ainsi on note le volume final c'est-à-dire le volume d'eau restant dans la première éprouvette. Le volume des pores est la différence des volumes initial et final.

La porosité s'exprime donc comme suite :

(3)

$$
\varepsilon \quad=\quad\left(\begin{array}{llll}
\mathrm{V}_{1} & - & \mathrm{V}_{2}
\end{array}\right) \quad / \mathrm{V}_{\mathrm{T}}
$$

$\varepsilon$ : macroporosité du garnissage

$\mathrm{V}_{1}$ : volume initial d'eau dans la première éprouvette $=1000 \mathrm{~mL}$

$\mathrm{V}_{2}$ : volume final d'eau dans la première éprouvette

$\mathrm{V}_{\mathrm{T}}$ : volume total de garnissage dans la deuxième éprouvette $=1000 \mathrm{~mL}$

\section{Diamètre moyen des particules}

Pour estimer le diamètre moyen des particules, nous avons recouru à la méthode d'éprouvette. Cette méthode consiste à remplir avec de l'eau une 
éprouvette de $100 \mathrm{~mL}$ jusqu'à la moitié. Ensuite on compte un nombre bien déterminé de noix de pain de singe ou de jujube que l'on introduit dans l'éprouvette. On note une augmentation de volume au niveau de l'éprouvette. Cette augmentation correspond au volume des noix introduites. Ainsi on peut déduire de cette augmentation de volume, premièrement, le volume unitaire moyen des noix et en deuxième lieu le diamètre moyen des noix.

$$
\mathrm{V}_{\mathrm{i}}=\left(\mathrm{V}_{1}-\mathrm{V}_{2}\right) / \mathrm{n}
$$

En considérant que les particules peuvent être assimilées à des sphères, on déduit à partir de la formule du volume de sphère le diamètre moyen.

$$
\begin{aligned}
\mathrm{V}_{\mathrm{i}}=\mathrm{V}_{\mathrm{S}} & =\frac{1}{6} \cdot \frac{1}{\pi \mathrm{dp}^{3}} \\
\mathrm{dp} & =\sqrt[3]{\frac{6 \mathrm{Vi}}{\pi}}
\end{aligned}
$$

Vi : volume unitaire de noix

$V_{S}$ : volume sphère

$\mathrm{V}_{1}$ : volume initial d'eau dans l'éprouvette $=100 \mathrm{~mL}$

$\mathrm{V}_{2}$ : volume final d'eau dans l'éprouvette

$\mathrm{n}:$ nombre de graines

dp : diamètre des particules

Il faut noter que, pour cette manipulation, nous avons choisi le nombre de graines $n=40$ en veillant à ce que le volume d'eau final ne dépasse pas $100 \mathrm{~mL}$. Ces paramètres physiques des deux supports de garnissage sont donnés dans le tableau I.

Tableau I : Caractéristiques physiques des noix de pain de singe et de jujube

\begin{tabular}{llllll}
\hline & $\begin{array}{l}\text { Masse } \\
\text { apparente }\left(\rho_{\text {ap }}\right) \text { en } \mathbf{g} / \mathbf{m L}\end{array}$ & $\begin{array}{l}\text { Masse volumique } \\
\text { réelle }\left(\rho_{\mathbf{r}}\right) \text { en } \mathbf{g} / \mathbf{m L}\end{array}$ & Porosité $(\varepsilon)$ & $\begin{array}{l}\text { Diamètre moyen } \\
\text { des } \\
(\mathbf{d p}) \text { en } \mathbf{m m}\end{array}$ \\
\hline $\begin{array}{l}\text { Noix de } \\
\text { de singe }\end{array}$ & 0,612 & 1,033 & 0,398 & 9.266 \\
Noix de jujube & 0,585 & 1,139 & 0,437 & 8,150 \\
\hline
\end{tabular}

\section{La colonne}

L'étude est réalisée sur une colonne transparente en verre retenue par un dispositif qui repose au plancher par des pieds. Le dispositif est constitué de tubes et de vis de serrage. La longueur de la colonne est de $1,5 \mathrm{~m}$ et son diamètre de $0,1 \mathrm{~m}$. Comme la plupart des colonnes garnies, celle utilisée dans cette étude est munie de support pour retenir les matériaux. Ce dernier repose sur le distributeur d'air qui le supporte. La colonne est surmontée par le haut, d'un ballon d'éclatement dans lequel est incorporé un support de tôle 
qui permet d'éviter la sortie de particules légères de garnissage. Mais aussi elle est munie en bas de colonne une entrée de la phase liquide qui est ici l'eau de robinet. En bas de colonne, il y a un distributeur d'air ; mais aussi, un robinet en bas de colonne, permet de vider la colonne à l'arrêt. La colonne est munie de 3 points de prise de pression reliés à un manomètre différentiel. Le schéma général du dispositif expérimental est présenté sur la figure 2.

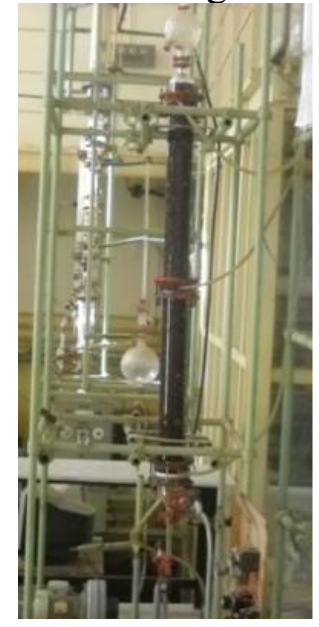

a .

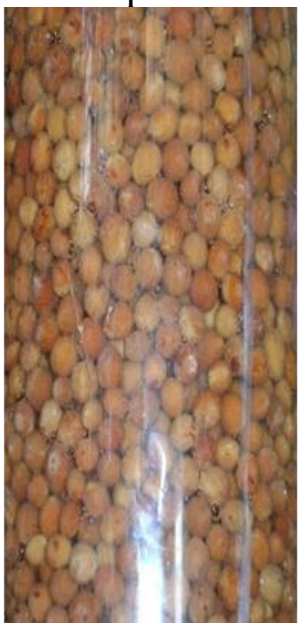

b.

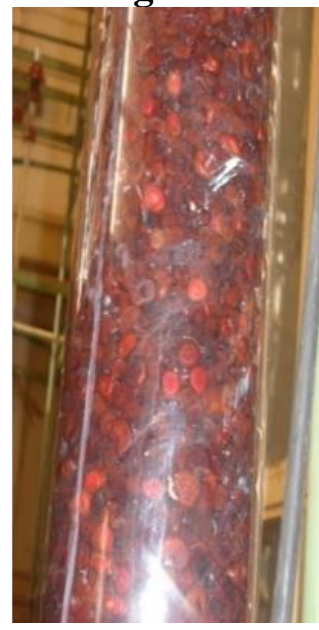

c .

Figure 2 : a-Illustration du bioréacteur; $\mathbf{b}$-Colonne garnie avec du jujube ; c-Colonne garnie avec du pain de singe

Mode opératoire des tests

\section{Méthode de détermination des pertes de charge}

Les pertes de charges sont mesurées à l'aide d'un manomètre différentiel comportant trois tubes en verre reliés à l'entrée, au milieu et à la sortie du garnissage respectivement.

Une fois le compresseur d'air actionné, on lance la pompe à eau qui fournit un débit d'eau que l'on définit. Par la suite, on attend quelques minutes pour que la colonne se remplisse et que le régime s'établisse. Ainsi nous lisons sur le manomètre la perte de charge en centimètres de colonne d'eau (cmCE).

Nous avons fait une série de mesures en variant les débits d'eau et d'air. Pour l'eau, nous avons travaillé dans la gamme de débit variant entre 36 et $96 \mathrm{~L} / \mathrm{h}$. Pour l'air la gamme de travail est de 35-190 L/h. Il faut noter que pour chaque débit d'air fixé les pertes de charge sont mesurées en variant les débits d'eau; il en est de même pour chaque débit d'eau fixé.

\section{Détermination de la rétention liquide dynamique}

Pour déterminer la rétention, il faut procéder à un remplissage de la colonne jusqu'à ce que le régime s'établisse. Une fois remplie, on arrête 
d'abord la pompe à eau puis l'arrivé d'air est fermée ; enfin la colonne est vidangée jusqu'au niveau de garnissage le plus bas. Le volume vidangé est recueilli et mesuré à l'aide d'une éprouvette graduée.

La rétention est ainsi déduite du volume d'eau vidangé à partir de la formule :

$$
\varepsilon_{\mathrm{LD}}=\frac{\mathrm{V}_{\mathrm{L}}}{\mathrm{V}_{\mathrm{T}}}
$$

Avec :

ELD : rétention liquide dynamique

$\mathrm{V}_{\mathrm{L}}$ : volume d'eau vidangé

$\mathrm{V}_{\mathrm{T}}$ : volume total du garnissage $\left(\mathrm{V}_{\mathrm{T}}=\pi \cdot \frac{\mathrm{D}^{2}}{4} \cdot \mathrm{H}_{\mathrm{lit}}\right)$

$\mathrm{D}$ : diamètre de la colonne

$\mathrm{H}_{\text {Lit }}$ : hauteur de garnissage

\section{Transfert de matière}

Pour évaluer le transfert de matière, nous allons déterminer le coefficient volumique de transfert de matière $\left(K_{\mathbf{L}} \mathbf{a}\right)$ en régime stationnaire et la capacité d'oxygénation $(\mathbf{C O})$ en passant par un bilan matière du sulfite de sodium $\left(\mathrm{Na}_{2} \mathrm{SO}_{3}\right)$ dans la colonne. Ainsi :

$$
\mathrm{TrO}_{2}=\left(\mathrm{K}_{\mathrm{La}}\right) . \quad(\Delta \mathrm{C}) . \quad\left(\mathrm{V}_{\mathrm{L}}\right) \quad \text { où } \quad \Delta \mathrm{C}=\frac{\mathrm{C}_{1}^{*}-\mathrm{C}_{2}^{*}}{\operatorname{Ln} \frac{\mathrm{C}_{1}^{*}}{\mathrm{C}_{2}^{*}}}
$$

(8)

$\mathrm{TrO}_{2}$ : quantité d'oxygène transférée en $\mathrm{g} / \mathrm{h}$.

$\mathrm{C}_{1}{ }^{*}$ : Concentration en oxygène dissous dans l'eau en équilibre avec l'air entrant

$\mathrm{C}_{2}{ }^{*}$ : Concentration en oxygène dissous dans l'eau en équilibre avec l'air sortant de la colonne

L'équation (8) ci-dessus permet de déterminer $\mathbf{K}_{\mathbf{L}} \mathbf{a}$. En d'autre terme, on peut évaluer le transfert de l'oxygène en matière de capacité d'oxygénation (voir équation 9).

(9)

$\mathrm{CO} \quad\left(\right.$ en $\left.\quad \mathrm{g} \cdot \mathrm{m}^{-3} \cdot \mathrm{h}^{-1}\right) \quad=\quad \mathrm{TrO}_{2} / \mathrm{Vg}$

$\mathrm{TrO}_{2}$ : quantité d'oxygène transférée en $\mathrm{g} / \mathrm{h}$;

$\mathrm{Vg}$ : volume occupé par le garnissage.

\section{Résultats et discussions}

\section{Pertes de charge}

Nous avons étudié l'évolution des pertes de charge en fonction des débits de gaz et de liquide. La figure 3 représente les courbes expérimentales des chutes de pression en fonction du débit d'air à des débits de liquide 
constants pour le pain de singe. Les débits de liquide de travail, ont été fixés à 72,84 , et $96 \mathrm{~L} / \mathrm{h}$.

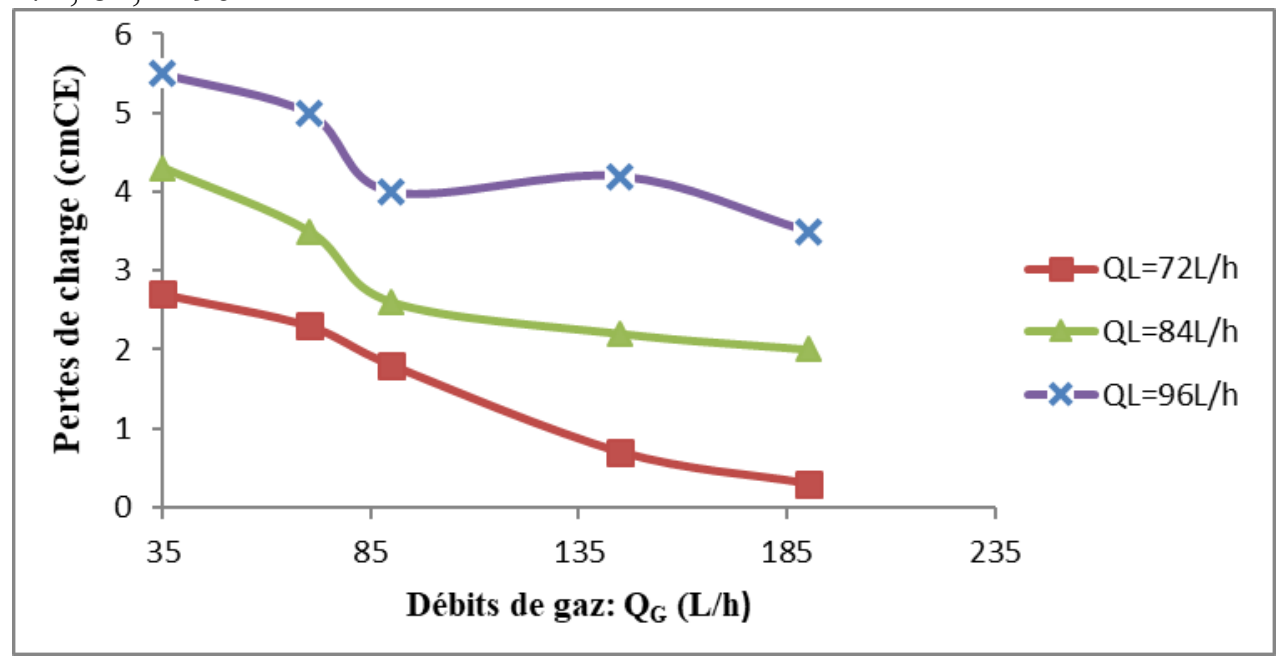

Figure 3 : Evolution des pertes de charge en fonction du débit d'air

Nous n'allons pas présentés les résultats obtenus pour les noix de jujube, car les mêmes tendances ont été observées. Ainsi, dans le cas du pain de singe, autant qu'avec le jujube, nous avons constaté que les pertes de charge varient faiblement en fonction des débits d'air. L'analyse des résultats obtenus montre l'influence du débit d'air sur les pertes de pression. En effet, elles diminuent avec l'augmentation du débit d'air. Pour un débit d'air de 85 $\mathrm{L} / \mathrm{h}$, on observe les chutes de pression sont 1,$8 ; 2,6$ et $4 \mathrm{cmCE}$ respectivement pour un débit de liquide de $72 ; 84$ et $94 \mathrm{~L} / \mathrm{h}$. De ce point de $\mathrm{vu}$, nous pouvons dire que les pertes de charge dépendent directement du débit et/ou la vitesse superficielle du liquide.

Cependant, le débit d'entrée de l'air est un facteur clé dans les procédés de biofiltration; d'une part, il conditionne le transfert d'oxygène dans la phase liquide. De plus, étant donné que les pertes de charge qui sont aussi des pertes d'énergie, ne dépendent pas trop du débit de gaz, ce débit peut être régulé selon la nécessité sans se soucier des coûts occasionnés par ces pertes dans le processus d'aération de la colonne.

Comme nous l'avons fait avec les débits d'air, nous allons maintenant varier les débits de liquide pour voir l'influence sur les pertes de charge. La figure 4 nous permet de voir l'effet de l'augmentation du débit de liquide sur les pertes de charge avec comme matériau de garnissage le pain de singe. La même tendance est obtenue avec le jujube. C'est pourquoi seul les résultats du pain de singe seront représentés. 


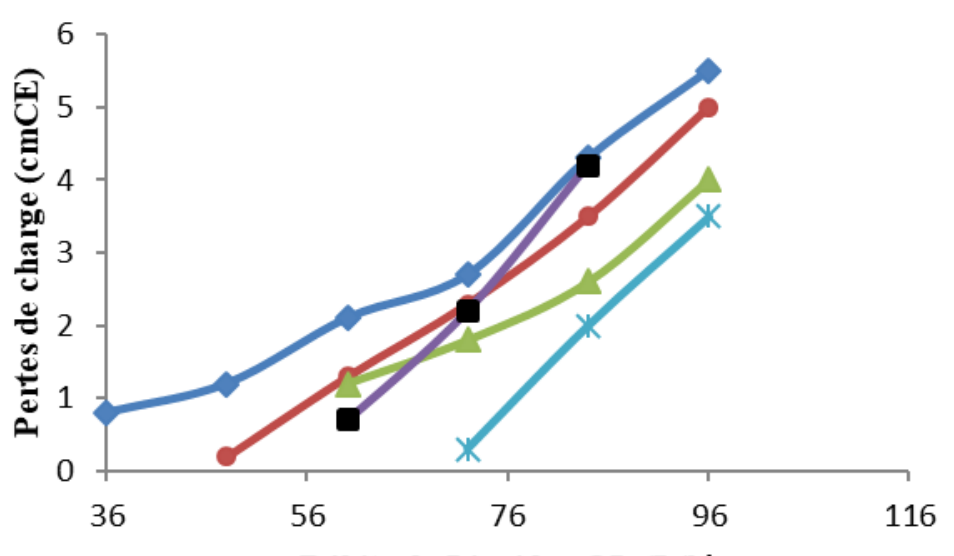

$-Q G=35 L / h$

$\longrightarrow Q G=70 L / h$

$-\mathrm{QG}=90 \mathrm{~L} / \mathrm{h}$

- $\mathrm{QG}=145 \mathrm{~L} / \mathrm{h}$

$\because Q G=190 \mathrm{~L} / \mathrm{h}$

Débits de Liquide: QL (L/h)

Figure 4 : Evolution des pertes de charge en fonction du débit d'eau

Contrairement à la représentation en fonction des débits de gaz, les pertes de charge varient considérablement en fonction des débits de liquide. Les pertes de charge croissent avec les débits de liquide. Les tendances observées à la figure 4 sont décrits avec précision par l'équation S. Ergun qui montre que les pertes de charge augmentent considérablement en fonction de la vitesse superficielle et/ou le débit de la phase liquide.

$$
\frac{\Delta P}{H}=A \frac{(1-\varepsilon)^{2}}{\varepsilon^{3}} \frac{\mu_{L}}{\left(\varphi_{p} d_{p}\right)^{2}} U_{L}+B \frac{1-\varepsilon}{\varepsilon^{3}} \frac{\rho_{L}}{\left(\varphi_{p} d_{p}\right)} U_{L}
$$

Les résultats obtenus au cours de nos expériences seront testés avec la corrélation de Turpin et Huntington (figures 5 et 4). Cette corrélation représente l'évolution du facteur de friction de type Fanning $\left(\mathrm{f}_{\mathrm{t}}\right)$ en fonction de $\operatorname{Ln}(\mathrm{z})$. Dans notre étude nous allons faire une linéarisation de la relation de Turpin et Huntington pour obtenir une nouvelle relation qui va tenir compte de nos conditions opératoires. 


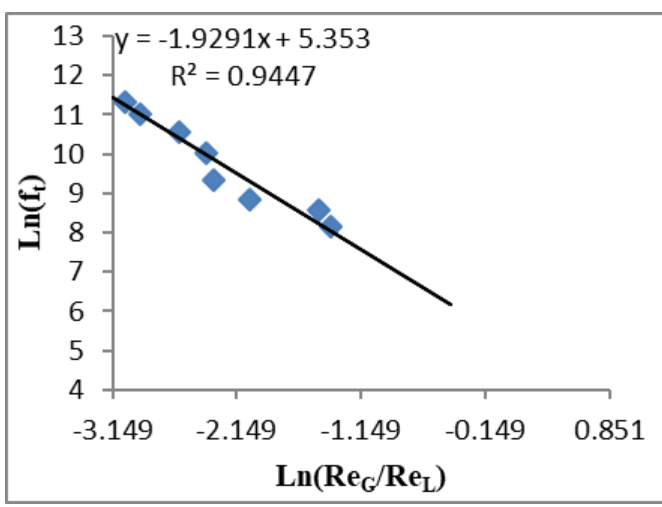

Figure 5 : Evolution du facteur de friction en fonction des nombres de Reynolds de liquide et de gaz pour le pain de singe

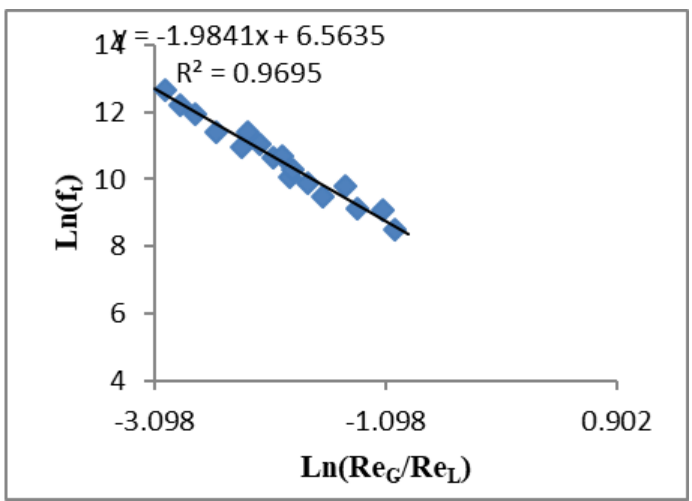

Figure 6 : Evolution du facteur de friction en fonction des nombres de Reynolds de liquide et de gaz pour le jujube

Les équations (11) et (12) obtenues à partir des données expérimentales sont en adéquation avec la corrélation de Turpin et Huntington (13). Nous pouvons supposer la thèse selon laquelle, les équations (11) et (12) peuvent être utilisées dans les travaux ultérieurs de notre bioréacteur à lit fixe tant que les conditions opératoires sont pareilles à celles que nous avions utilisées dans notre étude.

$\operatorname{Lnf}_{\mathrm{t}}$

(11)

$\mathrm{Lnf}_{\mathrm{t}}$

(12)

$\mathrm{Lnft}=$
$=$

$=$

8,0-1,12(Lnz)-0,0769(Lnz)
6,5635
$-1,9841(\operatorname{Lnz})$

$-1,9291(\mathrm{Lnz})$

0,0152(Lnz)

Avec $\mathrm{ft}=(\Delta \mathrm{P} / \Delta \mathrm{L}) \mathrm{LGde}_{\mathrm{e}} /\left(2 \mathrm{~V}_{\mathrm{SG}^{2}} \rho_{\mathrm{G}}\right), \mathrm{z}=\operatorname{Re}^{1,167} / \operatorname{Re}_{\mathrm{L}}{ }^{0,767}(\mu \mathrm{L} / \mu \mathrm{LQ})^{0.9}$ et $\mathrm{de}_{\mathrm{e}}=2 / 3$ $\mathrm{d}_{\mathrm{p}}(\varepsilon / 1-\varepsilon)$

\section{La rétention liquide dynamique}

La rétention liquide dynamique permet d'avoir une idée sur le transfert de matière gaz-liquide. Nous utiliserons la corrélation proposée par Stiegel et Shah (1977) pour tester nos résultats expérimentaux. Les résultats sont présentés dans le tableau II. 
Tableau II : Valeurs des rétentions liquides dynamiques pour le pain de singe et le jujube.

\begin{tabular}{|c|c|c|c|c|c|c|c|c|c|}
\hline \multirow{2}{*}{$\begin{array}{l}\mathbf{Q}_{\mathbf{L}} \\
(\mathbf{L} / \mathbf{h})\end{array}$} & \multirow{2}{*}{$\begin{array}{l}\mathbf{Q G}_{\mathbf{G}} \\
(\mathbf{L} / \mathbf{h})\end{array}$} & \multicolumn{2}{|c|}{ ELD expérimental } & \multirow{2}{*}{$\begin{array}{l}\mathbf{R e}_{\mathbf{L}} \\
\text { N.P.Singe }\end{array}$} & \multicolumn{2}{|r|}{ Reg } & \multicolumn{3}{|c|}{ ELD calculé } \\
\hline & & N.P.Singe & N.jujube & & N.jujube & N.P.Singe & N.jujube & N.P.Singe & N.jujube \\
\hline 36 & 70 & 0,227 & 0,339 & 13,201 & 11,615 & 1,460 & 1,285 & 0,231 & 0,347 \\
\hline 36 & 90 & 0,234 & 0,353 & 13,201 & 11,615 & 1,877 & 1,652 & 0,226 & 0,346 \\
\hline 36 & 145 & 0,214 & 0,345 & 13,201 & 11,615 & 3,0244 & 2,661 & 0,218 & 0,343 \\
\hline 48 & 70 & 0,229 & 0,348 & 17,601 & 15,487 & 1,460 & 1,284 & 0,229 & 0,351 \\
\hline 48 & 90 & 0,222 & 0,353 & 17,601 & 15,487 & 1,8772 & 1,652 & 0,224 & 0,349 \\
\hline 48 & 145 & 0,217 & 0,343 & 17,602 & 15,487 & 3,024 & 2,661 & 0,216 & 0,346 \\
\hline 60 & 70 & 0,226 & 0,356 & 22,002 & 19,358 & 1,460 & 1,285 & 0,228 & 0,354 \\
\hline 60 & 90 & 0,226 & 0,355 & 22,002 & 19,358 & 1,877 & 1,652 & 0,223 & 0,352 \\
\hline 60 & 145 & 0,214 & 0,346 & 22,002 & 19,358 & 3,024 & 2,661 & 0,214 & 0,349 \\
\hline
\end{tabular}

Les valeurs expérimentales et calculés de la rétention liquide dynamique du jujube sont plus élevées que celles du pain de singe. En effet, par exemple pour un débit de liquide constant de $36 \mathrm{~L} / \mathrm{h}$, la rétention liquide dynamique est 0,225 pour le pain de singe contre 0,345 pour le jujube. Cette tendance se manifeste pour toutes les données du tableau II. Ces résultats sont dus alors, du fait que la porosité du jujube est plus élevée que celle du pain de singe. Sachant que le transfert de matière est favorisé par de grandes rétentions, nous pouvons dire que dans ce cas, le jujube se présente comme meilleur garnissage pour les futures manipulations.

\section{Etude du transfert de matière et capacité d'oxygénation} Coefficient volumique de transfert de matière

Le transfert de matière gaz - liquide est caractérisé par le coefficient volumique de transfert de matière $\mathrm{K}_{\mathrm{L} a}$. Pour voir l'évolution des $\mathrm{K}_{\mathrm{La}}$ en fonction des vitesses d'air et de liquide, nous allons présenter les résultats sous forme de graphiques. La figure 7 représente l'évolution de $\mathrm{K}_{\mathrm{La}}$ en fonction de la vitesse superficielle de liquide à des débits de gaz constants pour le pain de singe. 


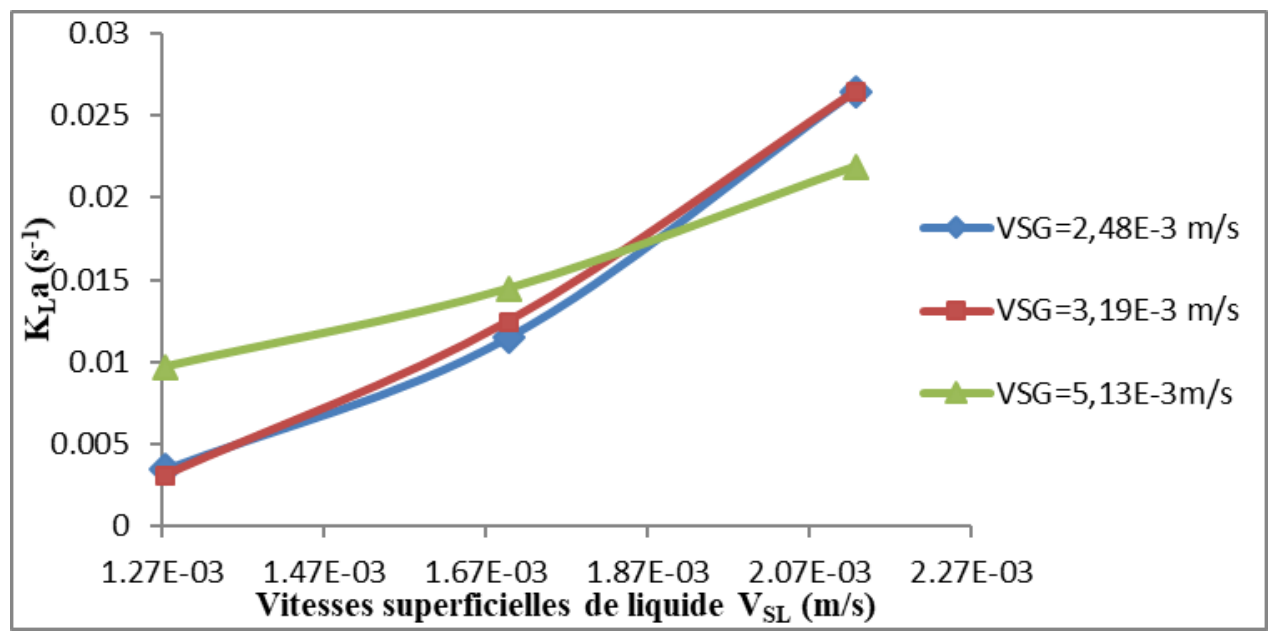

Figure 7 : Evolution de $\mathrm{K}_{\mathrm{L}}$ a en fonction de la vitesse superficielle de liquide

De la même façon que précédemment, la figure 8 montre l'évolution de $\mathrm{K}_{\mathrm{L}} \mathrm{a}$ en fonction des vitesses superficielles d'air à des débits de liquide constants avec comme garnissage le pain de singe.

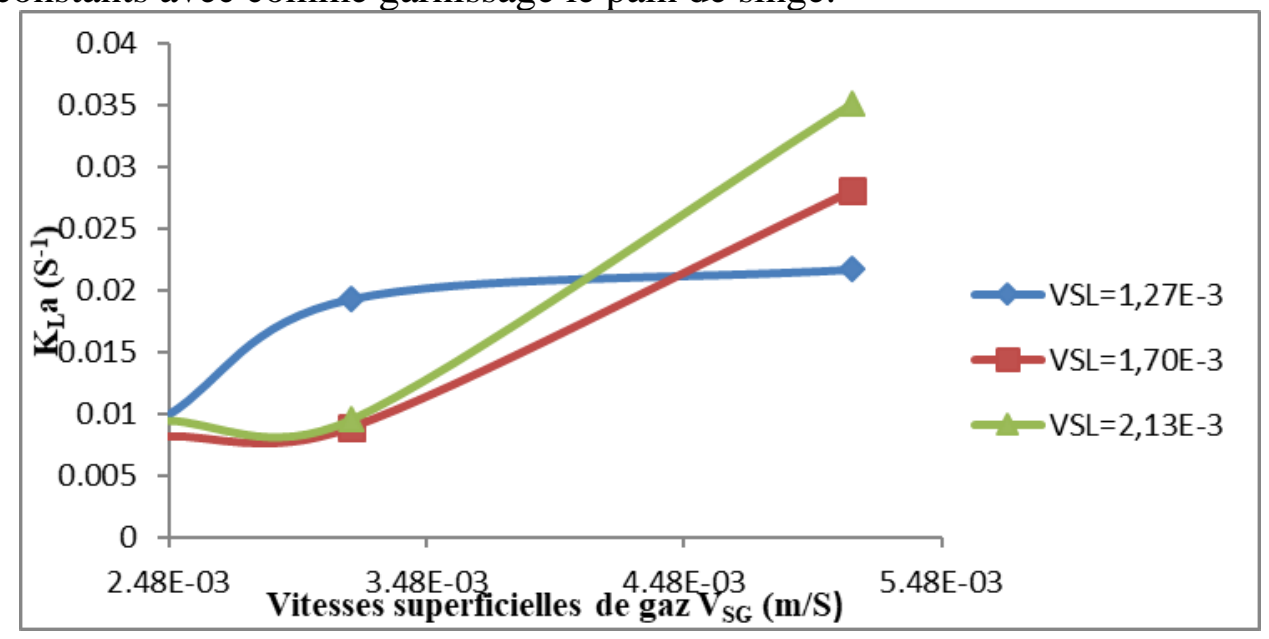

Figure 8 : Evolution de $\mathrm{K}_{\mathrm{L}}$ a en fonction de la vitesse superficielle de gaz

Sur la figure 7, nous constatons qu'il y'a une évolution du coefficient volumique de transfert de matière en fonction de la vitesse de liquide. Pour une même vitesse superficielle de gaz, l'augmentation de la vitesse superficielle de liquide entraîne une augmentation de la valeur du $K_{L}$ a. Maintenant en ce qui concerne la figure 8, nous voyons une évolution croissante du $\mathrm{K}_{\mathrm{La}}$ en fonction de la vitesse superficielle d'air. En effet, plus la vitesse d'air est élevée, plus le KLa caractéristique du transfert d'oxygène, est élevé. Pour le jujube aussi nous avons les mêmes évolutions. 
Nous avons également représenté les $\mathrm{K}_{\mathrm{L}} \mathrm{a}$ observés en fonction des $\mathrm{K}_{\mathrm{L}} \mathrm{a}$ calculés (voir figure 9 et 10).

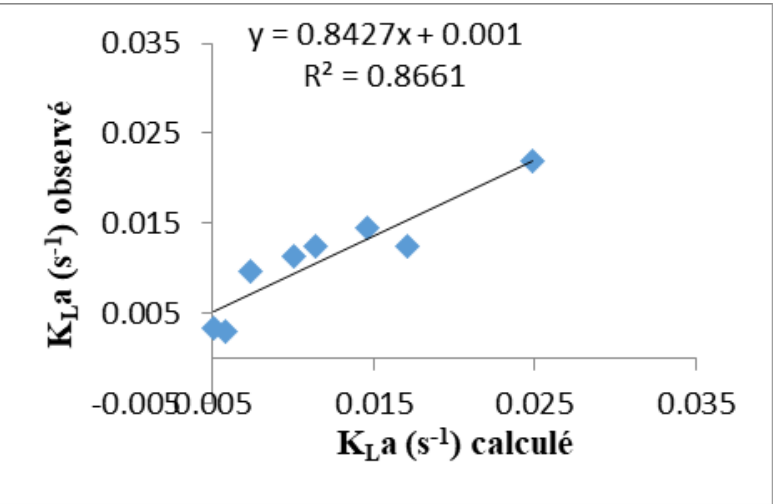

Figure 9 : Evolution du KLa observé en fonction du KLa calculé pour le pain de singe

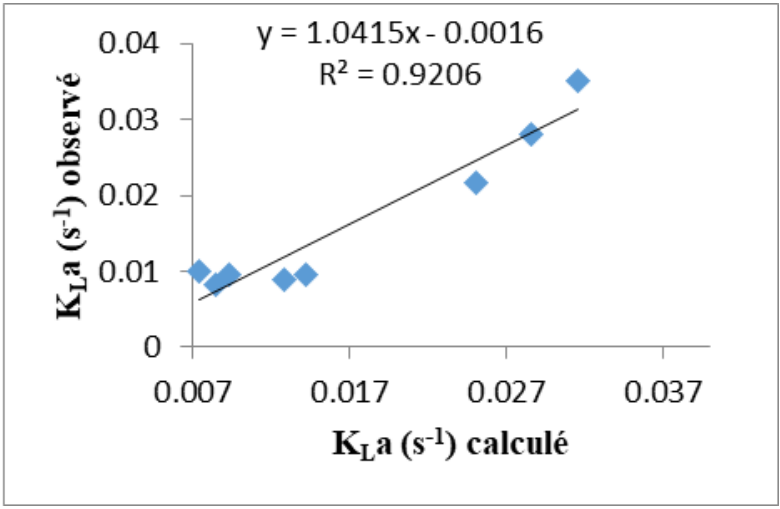

Figure 10 : Evolution du KLa observé en fonction du KLa calculé pour le jujube

Nous constatons (figure 9) qu'il y a une distribution plus ou moins bonne des résultats autour de la médiane. En effet en calculant l'erreur moyenne relative, nous avons $28,33 \%$. Par régression, nous avons pu établir pour le cas du pain de singe, une relation qui permettra de prédire les résultats expérimentaux du $\mathrm{K}_{\mathrm{L}}$.

$\mathrm{K}_{\mathrm{La}}$

$$
=
$$

$8,805 \cdot 10^{-6} \operatorname{Re}_{\mathrm{L}}$

${ }^{2,386} \mathrm{Re}_{\mathrm{G}}$

0,515

Il faut noter que l'équation (14) peut être utilisée pour la prévision des résultats expérimentaux dans un autre bioréacteur mais il faut au préalable travailler dans des conditions similaires aux nôtres.

La même démarche a été suivie pour le jujube pour pouvoir présenter une corrélation.

\section{Capacité d'oxygénation (CO)}

$\mathrm{Si}$ nous comparons les valeurs des coefficients volumiques de transfert de matière, c'est pour savoir parmi les deux matériaux quel est celui qui favorise mieux le transfert d'oxygène dans la phase liquide. Cette appréciation peut aussi être faite par la comparaison des capacités d'oxygénation. Ainsi avec les irrégularités notées avec les valeurs des KLa, nous allons effectuer la comparaison par les capacités d'oxygénation (CO). 
Tableau III : Valeurs des capacités d'oxygénation du pain de singe et du jujube

\begin{tabular}{|c|c|c|c|c|c|c|c|c|c|}
\hline \multirow{2}{*}{$\begin{array}{l}\mathbf{Q}_{\mathbf{L}} \\
(\mathbf{L} / \mathbf{h})\end{array}$} & \multirow{2}{*}{$\begin{array}{l}\mathbf{Q}_{\mathrm{G}} \\
(\mathrm{L} / \mathrm{h})\end{array}$} & \multicolumn{2}{|l|}{$\mathbf{V}_{\mathrm{SL}}(\mathbf{m} / \mathbf{s})$} & \multicolumn{2}{|l|}{$\mathbf{V}_{\text {SG }}(\mathrm{m} / \mathrm{s})$} & \multicolumn{2}{|l|}{$\mathrm{TrO}_{2}(\mathrm{~g} / \mathrm{h})$} & \multicolumn{2}{|c|}{$\operatorname{CO}\left(\mathrm{g} \cdot \mathrm{m}^{-3} \cdot \mathrm{h}^{-1}\right)$} \\
\hline & & N.P.Singe & N.jujube & N.P.Singe & N.jujube & N.P.Singe & N.jujube & N.P.Singe & N.jujube \\
\hline 36 & 70 & $1,27 \mathrm{E}-03$ & $1,27 \mathrm{E}-03$ & $2,48 \mathrm{E}-03$ & $2,48 \mathrm{E}-03$ & 0,27 & 1,116 & 22,918 & 94,729 \\
\hline 36 & 90 & $1,27 \mathrm{E}-03$ & $1,27 \mathrm{E}-03$ & $3,19 \mathrm{E}-03$ & 3,19E-03 & 0,252 & 2,124 & 21,390 & 180,290 \\
\hline 36 & 145 & $1,27 \mathrm{E}-03$ & 1,27E-03 & $5,13 \mathrm{E}-03$ & $5,13 \mathrm{E}-03$ & 0,702 & 2,304 & 59,587 & 195,569 \\
\hline 48 & 70 & $1,70 \mathrm{E}-03$ & $1,70 \mathrm{E}-03$ & $2,48 \mathrm{E}-03$ & $2,48 \mathrm{E}-03$ & 0,888 & 0,96 & 75,376 & 81,487 \\
\hline 48 & 90 & $1,70 \mathrm{E}-03$ & $1,70 \mathrm{E}-03$ & 3,19E-03 & $3,19 \mathrm{E}-03$ & 0,936 & 1,056 & 79,450 & 89,636 \\
\hline 48 & 145 & $1,70 \mathrm{E}-03$ & $1,70 \mathrm{E}-03$ & $5,13 \mathrm{E}-03$ & 5,13E-03 & 1,056 & 2,976 & 89,636 & 252,610 \\
\hline 60 & 70 & $2,13 \mathrm{E}-03$ & $2,13 \mathrm{E}-03$ & $2,48 \mathrm{E}-03$ & $2,48 \mathrm{E}-03$ & 0,96 & 1,14 & 81,4873 & 96,7662 \\
\hline 60 & 90 & $2,13 \mathrm{E}-03$ & $2,13 \mathrm{E}-03$ & $3,19 \mathrm{E}-03$ & $3,19 \mathrm{E}-03$ & 1,5 & 1,14 & 127,324 & 96,766 \\
\hline 60 & 145 & 2,13E-03 & 2,13E-03 & $5,13 \mathrm{E}-03$ & $5,13 \mathrm{E}-03$ & 1,56 & 3,75 & 132,417 & 318,309 \\
\hline
\end{tabular}

Dans ce tableau, nous pouvons constater que les capacités d'oxygénation obtenues avec le jujube sont plus élevées que celles du pain de singe. En outre, pour des débits constants de liquide de 36, 48 et $60 \mathrm{~L} / \mathrm{h}$, nous avons noté des valeurs de $\mathrm{CO}$ de 34,63 contre 156,86, 81,48 contre 141,14 et 113,74 contre 170,61 respectivement pour le pain de singe et le jujube. Or plus la capacité d'oxygénation est élevée plus le transfert est meilleur. S'il y a lieu d'un choix, par rapport au transfert d'oxygène, le garnissage le plus adapté est le jujube.

En résumé, nous venons de faire une comparaison entre les deux types de garnissage avec lesquels nous avons travaillés : les noix de pain de singe et de jujube. Ainsi, de manière globale, il est plus judicieux de choisir les noix de jujube comme garnissage. Par ailleurs, une comparaison de nos résultats pourrait être faite avec les travaux (Maldonado et al., 2008). Cependant il faut noter que ce choix sur le jujube n'exclut pas l'utilisation du pain de singe comme garnissage parce que comparé aux résultats obtenus dans la littérature, il donne de bons résultats.

\section{Conclusion}

L'objectif de ce travail a été d'effectuer une étude sur une nouvelle gamme de garnissage (noix de pain de singe et de jujube).

Dans la première partie nous avons eu à caractériser chaque matériau de garnissage par la détermination de paramètres physiques: les mases volumiques, la porosité et le diamètre des particules. La deuxième partie correspond à l'étude de l'hydrodynamique et du transfert d'oxygène. Dans l'étude hydrodynamique nous avons déterminé deux paramètres fondamentaux, à savoir : les pertes de charge ou pertes d'énergie ainsi que la rétention liquide dynamique. Pour ce qui est du transfert de matière, nous 
avons évalué les coefficients volumétriques de transfert de matière et déduire les capacités d'oxygénation.

L'étude comparative entre le pain de singe et le jujube révèle que pour tous les paramètres étudiés, le jujube fournit les meilleurs résultats et se présente donc comme meilleur garnissage dans les conditions de travail adoptées. Mais il faut souligner que le choix du jujube n'exclut pas le pain de singe comme garnissage.

Tableau IV : Récapitulatif des résultats expérimentaux

\begin{tabular}{|c|c|c|c|c|c|}
\hline Garnitures & $\Delta P(\mathrm{cmCE})$ & $\varepsilon_{L D}$ & $\mathbf{K}_{\mathrm{L}} \mathbf{a}\left(\mathbf{S}^{-1}\right)$ & $\mathrm{TrO}_{2}(\mathrm{~g} / \mathrm{h})$ & $\mathrm{CO}\left(\mathrm{g} \cdot \mathrm{m}^{-3} \cdot \mathrm{h}^{-1}\right)$ \\
\hline $\begin{array}{l}\text { Noix de Pain de } \\
\text { singe }\end{array}$ & 0,725 & 0,223 & $1,28 \cdot 10^{-2}$ & 0,902 & 70,282 \\
\hline Noix de jujube & 3,187 & 0,348 & $1,67 \cdot 10^{-2}$ & 1,840 & 163,674 \\
\hline
\end{tabular}

\section{References:}

1. Besagni, G., Inzoli, F., 2017. The effect of liquid phase properties on bubble column fluid dynamics: Gas holdup, flow regime transition, bubble size distributions and shapes, interfacial areas and foaming phenomena. Chemical Engineering Science 170, 270-296.

2. Dejaloud, A., Vahabzadeh, F., Habibi, A., 2018. Hydrodynamics and oxygen transfer characterization in a net draft tube airlift reactor with water-in-diesel microemulsion. Fuel Processing Technology 171, 265-276.

3. Garcia-Ochoa, F., Gomez, E., 2009. Bioreactor scale-up and oxygen transfer rate in microbial processes: an overview. Biotechnology advances 27, 153-176.

4. Jiang, L.-M., Garrido-Baserba, M., Nolasco, D., Al-Omari, A., DeClippeleir, H., Murthy, S., Rosso, D., 2017. Modelling oxygen transfer using dynamic alpha factors. Water research 124, 139-148.

5. Jokiel, M., Wagner, L.-M., Mansour, M., Kaiser, N.M., Zaehringer, K., Janiga, G., Nigam, K.D., Thevenin, D., Sundmacher, K., 2017. Measurement and simulation of mass transfer and backmixing behavior in a gas-liquid helically coiled tubular reactor. Chemical Engineering Science 170, 410-421

6. Maldonado, J.G., Bastoul, D., Baig, S., Roustan, M., Hébrard, G., 2008. Effect of solid characteristics on hydrodynamic and mass transfer in a fixed bed reactor operating in co-current gas-liquid up flow. Chemical Engineering and Processing: Process Intensification 47, 1190-1200

7. Palmerín-Carreño, D., Castillo-Araiza, C., Rutiaga-Quiñones, O., Verde-Calvo, J., Huerta-Ochoa, S., 2016. Kinetic, oxygen mass transfer and hydrodynamic studies in a three-phase stirred tank 
bioreactor for the bioconversion of (+)-valencene on Yarrowia lipolytica $2.2 \mathrm{ab}$. Biochemical engineering journal 113, 37-46.

8. Pan, A., Xie, M., Xia, J., Chu, J., Zhuang, Y., 2018. Gas-liquid mass transfer studies: The influence of single-and double-impeller configurations in stirred tanks. Korean Journal of Chemical Engineering 35, 61-72

9. Parisien, V., Pjontek, D., McKnight, C.A., Wiens, J., Macchi, A., 2017. Impact of catalyst density distribution on the fluid dynamics of an ebullated bed operating at high gas holdup conditions. Chemical Engineering Science 170, 491-500.

10. Park, J.H., Lee, M., Moriyama, K., Kim, M.H., Kim, E., Park, H.S., 2018. Adequacy of effective diameter in predicting pressure gradients of air flow through packed beds with particle size distribution. Annals of Nuclear Energy 112, 769-778.

11. Pavlišič, A., Pohar, A., Likozar, B., 2018. Comparison of computational fluid dynamics (CFD) and pressure drop correlations in laminar flow regime for packed bed reactors and columns. Powder Technology 328, 130-139.

12. Sharma, D., Taylor-Edmonds, L., Andrews, R.C., 2018. Comparative assessment of ceramic media for drinking water biofiltration. Water research 128, 1-9.

13. Thobie, C., Gadoin, E., Blel, W., Pruvost, J., Gentric, C., 2017. Global characterization of hydrodynamics and gas-liquid mass transfer in a thin-gap bubble column intended for microalgae cultivation. Chemical Engineering and Processing: Process Intensification 122, 76-89.

14. Xu, Y., Zhu, N., Sun, J., Liang, P., Xiao, K., Huang, X., 2017. Evaluating oxygen mass transfer parameters for large-scale engineering application of membrane bioreactors. Process Biochemistry 60, 13-18.

15. Zhang, J., Teixeira, A.R., Kögl, L.T., Yang, L., Jensen, K.F., 2017. Hydrodynamics of gas-liquid flow in micropacked beds: Pressure drop, liquid holdup, and two-phase model. AIChE Journal 63, 46944704 